\title{
BMJ Open Effectiveness of exercise for osteosarcopenia in older adults: a systematic review protocol
}

\author{
Guilherme Elias Vinícius-Souza (D) , ${ }^{1}$ Matias Noll (D) ,1,2,3 \\ Erika Aparecida Silveira (D) ${ }^{1}$
}

To cite: Vinícius-Souza GE, Noll M, Silveira EA.

Effectiveness of exercise

for osteosarcopenia in older adults: a systematic review protocol. BMJ Open 2021;11:e045604. doi:10.1136/ bmjopen-2020-045604

- Prepublication history and additional supplemental material for this paper are available online. To view these files, please visit the journal online (http://dx.doi.org/10.1136/ bmjopen-2020-045604).

Received 06 0ctober 2020 Accepted 20 April 2021

D) Check for updates

(c) Author(s) (or their employer(s)) 2021. Re-use permitted under CC BY-NC. No commercial re-use. See rights and permissions. Published by BMJ.

${ }^{1}$ Postgraduate Program in Health Sciences, Faculdade de Medicina, Universidade Federa de Goias, Goiania, Goiás, Brazil ${ }^{2}$ Public Health, Instituto Federal Goiano, Ceres, Goiás, Brazil ${ }^{3}$ University of Southern Denmark, Odense, Syddanmark, Denmark

\section{Correspondence to}

Guilherme Elias Vinícius-Souza; gui.souza0607@gmail.com and Erika Aparecida Silveira; erikasil@terra.com.br

\section{ABSTRACT}

Introduction 0steosarcopenia is defined as the concomitant occurrence of sarcopenia and osteopenia or osteoporosis. Older adults with this syndrome have a greater fragility and mortality risk compared with those without these conditions. Based on separate interventions with individuals with sarcopenia and osteoporosis, exercise has been recommended as a treatment for osteosarcopenia. However, there is no evidence of its efficacy. Our objective is to identify whether physical exercise can improve osteosarcopenia in older adults and lead to good health outcomes.

Methods and analysis We will perform a systematic review in the following databases: PubMed, Embase, Cochrane and Scopus. The criterion of inclusion will be clinical trials involving physical exercise interventions in older adults diagnosed with osteosarcopenia. To assess the risk of bias, the Grading of Recommendations, Assessment, Development and Evaluation and Downs and Black tools will be used. For each search result, the quality of the evidence will ultimately receive one of four grades: high, moderate, low or very low. The outcome of this study is to demonstrate the effectiveness of physical exercise in improving the parameters that lead to the diagnosis of osteosarcopenia (bone mineral density, quality of muscle mass, muscle strength and physical function) in older adults. The possibility of meta-analysis will be assessed according to the homogeneity of the studies, using the methods of fixed or random effects. Sensitivity analyses will be performed, and the funnel plot will be used to assess publication bias. The proposed statistical analyses will be performed using STATA software, V.14.0.

Ethics and dissemination The results of the systematic review will be disseminated via publication in a peerreviewed journal and presented at a relevant conference. As we will not use individual patient data, ethical approval is not required.

Trial registration number CRD42020215659.

\section{INTRODUCTION}

A recently defined syndrome, ${ }^{1}$ osteosarcopenia, refers to the presence of sarcopenia plus osteopenia or osteoporosis in the same individual. ${ }^{2}$ The diagnosis of osteopenia/ osteoporosis requires an assessment of bone mineral density (BMD) through dual-energy X-ray absorptiometry (DEXA). ${ }^{34}$ Sarcopenia,

\section{Strengths and limitations of this study}

- Four important databases (PubMed (National Library of Medicine), Embase, Cochrane and Scopus) will be analysed.

- Our investigation will seek to determine the duration, frequency, intensity and type of physical exercise that is most appropriate for preventing and treating osteosarcopenia.

- There may be high heterogeneity owing to the various diagnostic criteria and cut-off points for sarcopenia.

- There will be no language bias as our search will not place restrictions on language of publication.

- The results will contribute to the development of health prevention and promotion programmes and may fill the gap pertaining to accurate physical exercise prescription in older adults with osteosarcopenia.

on the contrary, is characterised by low muscle strength, with diagnosis confirmed through the detection of low muscle quality and severity classified based on the weakness of physical performance. ${ }^{5}$

The prevalence of osteosarcopenia in older adults varies by country. A study in Germany reported a prevalence of $28 \%,{ }^{6}$ while in a study of Australian community dwellers, the rate was $37 \%{ }^{7}$ In addition, when compared with older adults with isolated conditions, that is, sarcopenia, osteopenia or osteoporosis, those with osteosarcopenia have more fractures $^{8}$ and a $15.1 \%$ higher risk of mortality. ${ }^{9}$

Physical exercise (PE) has been recommended as a preventive strategy or nonpharmacological therapeutic approach for osteosarcopenia. ${ }^{10-12}$ However, PE is prescribed in the context of isolated conditions, failing to consider osteosarcopenia as a unique condition that encompasses sarcopenia plus osteopenia/osteoporosis. ${ }^{11} 1314$ While there is a consensus in the literature on the effectiveness of PE for older adults with sarcopenia and osteoporosis, ${ }^{15-18}$ few 
studies have explored the efficacy of PE in people with osteosarcopenia.

Regarding osteosarcopenia in older adults, we were unable to identify any literature review addressing the effects of regular engagement in PE. Therefore, our aim is to develop a systematic review of the literature that can answer the following question: 'What are the influences of regular PE practice on osteosarcopenia in older adults?' The results of this study may promote the application of PE as a form of nonpharmacological or complementary therapy for the prevention or treatment of osteosarcopenia.

\section{METHODS AND ANALYSIS}

This systematic review will be conducted according to the criteria of the Preferred Reporting Items for Systematic Review and Meta-Analysis Protocols (PRISMA-P). ${ }^{19}{ }^{20} \mathrm{We}$ adopted the 'PICO' structure and defined it as follows: 'P' corresponds to older adults, 'I' to physical PE, 'C' to the absence of $\mathrm{PE}$ and ' $\mathrm{O}$ ' to osteosarcopenia.

\section{Eligibility criteria}

Controlled, randomised, blind or open clinical trials, without language and publication period restrictions, that perform any type of minimum 4-week PE intervention with older adults (aged $\geq 65$ years) of both sexes diagnosed with concomitant osteopenia/osteoporosis and sarcopenia will be included.

We will consider individuals with osteopenia with a T-score $\leq 1$ in measurements performed by DEXA on the femoral neck, lumbar spine or total hip, according to the WHO's diagnostic criteria for BMD. ${ }^{3}$

Sarcopenia has various definitions and diagnostic criteria. $^{521}$ Therefore, the criteria and cut-off points already proposed in studies that perform musculoskeletal measurements will be used, ${ }^{52-23}$ and studies with older adults with reduced muscle strength and mass will be included. Studies carried out with older adults with osteosarcopenic obesity will also be included in this review.

However, studies with hospitalised older adults, with a focus on specific conditions, for example, stroke, will be excluded. We will also exclude animal studies, observational studies, opinion articles, editorials, narrative reviews, case series and comments and duplicate studies. Publications that meet the inclusion criteria but for which the results are unavailable even after consulting the authors will be excluded.

\section{Database search}

Our search will be conducted in four databases (PubMed (National Library of Medicine), Embase, Cochrane and Scopus). In addition, the GreyNet International platform and Google Scholar will be used to locate grey literature. To complement the search and ensure the saturation of the literature, the references of the selected articles will also be considered.
The search terms have been selected using Medical Subject Headings (MeSH) for the PubMed database. Search: ((Osteosarcopenia [Title/Abstract]) OR (Sarcoosteopenia [Title/Abstract]) OR (Osteo-sarcopenia [Title/Abstract]) OR (Osteosarcopenic [Title/Abstract]) AND ( ( ( (exercise [MeSH Terms]) OR (exercise [Title/ Abstract])) OR (physical activity [Title/Abstract])) OR (physical activities [Title/Abstract])) OR ("Physical Fitness" [Title/Abstract] $)))$ AND $(((()(($ aged [MeSH Terms]) OR (aged [Title/Abstract])) OR (elderly [Title/ Abstract])) OR (older adult [Title/Abstract])) OR (older adults [Title/Abstract])) OR (elder [Title/Abstract])) OR (Ageing [Title/Abstract])) OR (aging [Title/ Abstract])). The same search model will be adapted to Embase, Cochrane, and Scopus databases (online supplemental file 1). Searches will be carried out in April 2021.

\section{Review process}

The search for articles will be carried out by two independent researchers (GEV-S and $\mathrm{MN}$ ) and a third senior reviewer (EAS). The inclusion of articles will be based on a reading of the titles, and, later, the abstracts. Finally, the complete content will be analysed for inclusion.

After executing the search strategy, articles will be collated, and duplicates will be removed using Mendeley software. Then, two reviewers (GEVS and MN) will independently screen the titles and abstracts of all articles identified in the literature search. Disagreements regarding inclusion will be discussed and resolved by a third reviewer (EAS). Both reviewers will perform the screening process using Rayyan software. ${ }^{24}$ Inter-rater reliability for individual component ratings will be determined by calculating the percentage of agreement and the Cohen's Kappa coefficient. ${ }^{25}$ The remaining articles will be read in full and evaluated to determine their eligibility based on the inclusion and exclusion criteria. Finally, eligible articles will be included in the systematic review. The entire process of selecting articles for the systematic review will be finalised by the end of September 2021 .

According to PRISMA-P recommendations, we will prepare a flowchart with information about the screening of studies, reasons for inclusion and exclusion and recording and viewing of this process.

\section{Data extraction and study quality assessment}

Data extraction and study quality assessment will be performed by two reviewers (GEVS and MN); disagreements will be resolved by a third reviewer (EAS). To extract data from an article, a standardised form prepared by the authors will be used. The following aspects will be considered: author/year of publication, place of study, age group studied, sample size, intervention performed, time of intervention and main results.

Risk of bias will be assessed using the 27-item Downs and Black instrument. ${ }^{26} \mathrm{~A}$ score will be calculated for each manuscript (0-27 points) and scores above $70 \%$ will be used to define low risk of bias. 
The Grading of Recommendations, Assessment, Development and Evaluation ${ }^{27}$ system will be used to assess the quality of evidence. For each research result, the quality of the evidence will be classified as one of the following: high, moderate, low or very low. ${ }^{28}$

We will also analyse whether the authors of the included studies have addressed the impact of possible conflicts of interest and information regarding ethical approval. ${ }^{29} \mathrm{We}$ intend to finalise the data extraction process and evaluate the quality of the studies at the beginning of April 2021.

\section{Data analysis}

The outcome of this study is improvement in osteosarcopenia (BMD, appendicular muscle mass, muscle strength and function) in older adults. For subgroup analysis, we will consider the following variables: sex, age group and body mass index. We will group the results in metaanalysis of random effects and these will be reported using a forest plot to show the grouped effect of the findings. In addition, we will use a funnel plot for data accuracy assessment. ${ }^{30}$ The percentage of variability attributable to heterogeneity will be estimated using the I2 test. I2 values of $<40,40-60,60-90$ and $>90 \%$ correspond, respectively, to 'not important', 'moderate', 'substantial' and 'considerable' levels of heterogeneity. ${ }^{30}$ The proposed statistical analyses will be performed using STATA software, V.14.0.

\section{Patient and public involvement \\ No patients involved.}

\section{DISCUSSION}

PE prescriptions are generally based on specific recommendations for osteoporosis and sarcopenia. ${ }^{10} 11$ This is why our investigation will seek to determine the specific duration, frequency, intensity and type of PE that is most appropriate for preventing and treating osteosarcopenia. We will seek to broaden the research by addressing the potential differences associated with varying exercise modalities: low or high-impact aerobics, resistance exercise, balance exercises, combined exercises and wholebody vibration.

Through this protocol, it will be possible to conduct a systematic review and meta-analysis to elucidate the type of $\mathrm{PE}$ that is most effective for the prevention and/or treatment of osteosarcopenia in older adults. At present, we are not aware of a published systematic review on this topic. Consequently, the results may hold important implications for the field of gerontology. At the conclusion of this project, we aim to clarify the influence of $\mathrm{PE}$ on the parameters of osteosarcopenia, to trace paths of recommendations for possible therapeutic practices and to identify the need for new studies.

\section{Ethics and dissemination}

The results of the systematic review will be disseminated via publication in a peerreviewed journal and presented at a relevant conference. As the data will not includeindividual patient data, ethical approval is not required.

AcknowledgmentsThe authors are grateful to the Instituto Federal Goiano for support and the Coordination for the Improvement of Higher Education Personnel (Coordenação de Aperfeiçoamento Pessoal de Nível Superior - CAPES) (http:// www.capes.gov.br) for providing fellowship to GEV-S. Also, we are grateful to the Postgraduate Program in Health Sciences, Faculty of Medicine, Federal University of Goias for the APC support.

Contributors GEV-S and EAS conceived the study idea. GEV-S, EAS and MN contributed to the design of the systematic review. GEV-S, EAS and MN contributed to the data analysis plan. All authors contributed to the writing and editing of the manuscript and approved the final manuscript.

Funding The authors have not declared a specific grant for this research from any funding agency in the public, commercial or not-for-profit sectors.

Competing interests None declared.

Patient consent for publication Not required.

Provenance and peer review Not commissioned; externally peer reviewed.

Supplemental material This content has been supplied by the author(s). It has not been vetted by BMJ Publishing Group Limited (BMJ) and may not have been peer-reviewed. Any opinions or recommendations discussed are solely those of the author(s) and are not endorsed by BMJ. BMJ disclaims all liability and responsibility arising from any reliance placed on the content. Where the content includes any translated material, BMJ does not warrant the accuracy and reliability of the translations (including but not limited to local regulations, clinical guidelines, terminology, drug names and drug dosages), and is not responsible for any error and/or omissions arising from translation and adaptation or otherwise.

Open access This is an open access article distributed in accordance with the Creative Commons Attribution Non Commercial (CC BY-NC 4.0) license, which permits others to distribute, remix, adapt, build upon this work non-commercially, and license their derivative works on different terms, provided the original work is properly cited, appropriate credit is given, any changes made indicated, and the use is non-commercial. See: http://creativecommons.org/licenses/by-nc/4.0/.

\section{ORCID iDs}

Guilherme Elias Vinícius-Souza http://orcid.org/0000-0001-6401-9200

Matias Noll http://orcid.org/0000-0002-1482-0718

Erika Aparecida Silveira http://orcid.org/0000-0002-8839-4520

\section{REFERENCES}

1 Binkley N, Buehring B. Beyond FRAX: it's time to consider "sarcoosteopenia". J Clin Densitom 2009;12:413-6.

2 Hirschfeld HP, Kinsella R, Duque G. Osteosarcopenia: where bone, muscle, and fat collide. Osteoporos Int 2017;28:2781-90 http://link. springer.com/

3 WHO. Who scientific group on the assessment of osteoporosis at primary health care level summary meeting report 2004.

4 Cosman F, de Beur SJ, LeBoff MS. Erratum to: Clinician's guide to prevention and treatment of osteoporosis. Osteoporos Int 2015. [Epub ahead of print: Available from] http://link.springer.com/ doi:10.1007/s00198-015-3037-x

5 Cruz-Jentoft AJ, Bahat G, Bauer J, et al. Sarcopenia: revised European consensus on definition and diagnosis. Age Ageing 2019;48:16-31.

6 Drey M, Sieber CC, Bertsch T, et al. Osteosarcopenia is more than sarcopenia and osteopenia alone. Aging Clin Exp Res 201610.1007/ s40520-015-0494-1. [Epub ahead of print: Available from] http://link. springer.com/

7 Huo YR, Suriyaarachchi P, Gomez F. Phenotype of osteosarcopenia in older individuals with a history of falling. J Am Med Dir Assoc 2015. [Epub ahead of print: Available from] https://linkinghub. elsevier.com/retrieve/pii/S1525861014006963 doi:10.1016/j. jamda.2014.10.018

8 Wang Y-J, Wang Y, Zhan J-K, et al. Sarco-Osteoporosis: prevalence and association with frailty in Chinese community-dwelling older adults. Int J Endocrinol 201510.1155/2015/482940. [Epub ahead of print: Available from] http://www.hindawi.com/journals/ije/2015/ 482940/ 
9 Yoo Jl, Kim H, Ha YC, et al. Osteosarcopenia in patients with hip fracture is related with high mortality. J Korean Med Sci 2018;33:e27 https://jkms.org/DOlx.php?id=10.3346/jkms.2018.33.e27

10 Paintin J, Cooper C, Dennison E. Osteosarcopenia. Br J Hosp Med 2018;79:253-8.

11 Fatima M, Brennan-Olsen SL, Duque G. Therapeutic approaches to osteosarcopenia: insights for the clinician. Ther Adv Musculoskelet Dis 2019;11:1759720X1986700.

12 Kemmler W, Kohl M, Fröhlich M, et al. Effects of high-intensity resistance training on osteopenia and sarcopenia parameters in older men with Osteosarcopenia-One-Year results of the randomized controlled Franconian osteopenia and sarcopenia trial (frost). J Bone Miner Res 2020;35:1634-44.

13 Hassan EB, Duque G. Osteosarcopenia: A new geriatric syndrome The role of the Kynurenine Pathway in Osteoblastogenesis View project [Internet], 2017. Available: https://www.researchgate.net/ publication/320974030

14 Kirk B, Al Saedi A, Duque G. Osteosarcopenia: a case of geroscience. Aging Med 201910.1002/agm2.12080. [Epub ahead of print: Available from] https://onlinelibrary.wiley.com/doi/abs/

15 Vlietstra L, Hendrickx W, Waters DL. Exercise interventions in healthy older adults with sarcopenia: a systematic review and meta-analysis. Australas J Ageing 201810.1111/ajag.12521. [Epub ahead of print: Available from] http://doi.wiley.com/

16 Kirk B, Mooney K, Amirabdollahian F, et al. Exercise and DietaryProtein as a countermeasure to skeletal muscle weakness: Liverpoo hope university - sarcopenia aging trial (LHU-SAT). Front Physiol 2019;10.

17 Gonzalo-Encabo P, McNeil J, Boyne DJ. Dose-response effects of exercise on bone mineral density and content in post-menopausal women. Scandinavian Journal of Medicine \& Science in Sports 2019;13443.

18 Matzkin EG, DeMaio M, Charles JF, et al. Diagnosis and treatment of osteoporosis. J Am Acad Orthop Surg 201910.5435/ JAAOS-D-18-00600. [Epub ahead of print: Available from] http:// journals.Iww.com/00124635-201910150-00006

19 Moher D, Stewart L, Shekelle P. Implementing PRISMA-P: recommendations for prospective authors. Syst Rev 2016;5 doi:10.1186/s13643-016-0191-y. [Epub ahead of print: Available from] http://www.systematicreviewsjournal.com/content/5/1/15

20 Moher D, Shamseer L, Clarke M, et al. Preferred reporting items for systematic review and meta-analysis protocols (PRISMA-P) 2015 statement. Syst Rev 2015;4:1.
21 Cruz-Jentoft AJ, Baeyens JP, Bauer JM, et al. Sarcopenia: European consensus on definition and diagnosis: report of the European Working group on sarcopenia in older people. Age Ageing 201010.1093/ageing/afq034. [Epub ahead of print: Available from] https://academic.oup.com/ageing/article-lookup/doi/

22 Chen L-K, Liu L-K, Woo J, et al. Sarcopenia in Asia: consensus report of the Asian Working group for sarcopenia. J Am Med Dir Assoc 201410.1016/j.jamda.2013.11.025. [Epub ahead of print: Available from] https://linkinghub.elsevier.com/retrieve/pii/ S1525861013006671

23 Tanimoto Y, Watanabe M, Sun W, et al. Association between muscle mass and disability in performing instrumental activities of daily living (IADL) in community-dwelling elderly in Japan. Arch Gerontol Geriatr 201210.1016/j.archger.2011.06.015. [Epub ahead of print: Available from] https://linkinghub.elsevier.com/retrieve/pii/ S0167494311001701

24 Ouzzani M, Hammady H, Fedorowicz Z, et al. Rayyan-a web and mobile APP for systematic reviews. Syst Rev 2016;5. doi:10.1186/ s13643-016-0384-4. [Epub ahead of print: Available from] http://syst ematicreviewsjournal.biomedcentral.com/articles/

25 Stefani L, Galanti G, Padulo J, et al. Sexual activity before sports competition: a systematic review. Front Physiol 2016;7:246.

26 Downs SH, Black N. The feasibility of creating a checklist for the assessment of the methodological quality both of randomised and non-randomised studies of health care interventions. J Epidemiol Community Health 199810.1136/jech.52.6.377. [Epub ahead of print: Available from] http://jech.bmj.com/cgi/doi/

27 Balshem H, Helfand M, Schünemann HJ, et al. Grade guidelines: 3. rating the quality of evidence. J Clin Epidemiol 201110.1016/j. jclinepi.2010.07.015. [Epub ahead of print: Available from] https:// linkinghub.elsevier.com/retrieve/pii/S089543561000332X

28 Grading quality of evidence and strength of recommendations. BMJ 2004;328. [Epub ahead of print: Available from] http://www.bmj.com/ lookup/doi/

29 Elia N, von Elm E, Chatagner A, et al. How do authors of systematic reviews deal with research malpractice and misconduct in original studies? A cross-sectional analysis of systematic reviews and survey of their authors. BMJ Open 2016;6:e010442.

30 Higgins J, Thomas J. Cochrane Handbook for systematic reviews of interventions. Cochrane Collab 2019. 